\title{
Identification and functional study of osteosarcoma metastasis marker genes
}

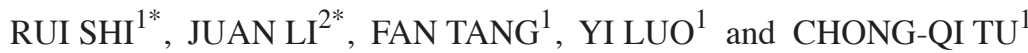 \\ ${ }^{1}$ Department of Orthopedics, West China Hospital, Sichuan University; ${ }^{2}$ Department of Pulmonary Tumors, \\ Sichuan Cancer Hospital, Chengdu, Sichuan 610041, P.R. China
}

Received September 19, 2014; Accepted May 7, 2015

DOI: $10.3892 / \mathrm{ol} .2015 .3444$

\begin{abstract}
To date, osteosarcoma metastasis genes, which are key for accurate initial diagnosis of the disease, have not been well identified. In the present study, osteosarcoma samples with and without metastasis were collected from 31 patients. Specific complementary DNA subtraction techniques were used to identify the osteosarcoma metastasis transcripts, which are responsible for the metastasis of osteosarcoma. The specific differentially expressed transcripts were identified by Basic Local Alignment Search Tool analysis and the results were validated by immunoblotting. Specifically, ezrin and $\beta 4$ integrin were employed as markers to detect osteosarcoma metastasis in the initial stages. The results of the present study indicated that the two transcripts, ezrin and $\beta 4$ integrin, were highly expressed in patients with osteosarcoma metastasis, and concluded that these were osteosarcoma metastasis genes. These results indicate that $\beta 4$ integrin and/or ezrin may be used as a novel marker for the detection of osteosarcoma metastasis in the initial stages.
\end{abstract}

\section{Introduction}

Metastasis is a complex process describing the spread of cancerous cells from the primary tumor site to other organs. Osteosarcoma is the most common primary bone tumor amongst children and young adults (1). The most prevalent locations for osteosarcoma are the distal femoral, femur and proximal tibial metaphyses (2). It was reported that in $\sim 50 \%$ of cases, osteosarcoma was localized to the knee region (3). Patients with bone metastases experience severe bone pain, spinal cord compression and osteolysis, which reduce the structural integrity of bone and results in increased risk of fracture (4). Osteosarcoma

Correspondence to: Dr Chong-Qi Tu, Department of Orthopedics, West China Hospital, Sichuan University, 37 Guoxue Lane, Chengdu, Sichuan 610041, P.R. China

E-mail: tuchongqi61@hotmail.com

${ }^{*}$ Contributed equally

Key words: osteosarcoma, metastasis, osteosarcoma metastasis genes, immunoblotting progression results in cell migration from the primary tumor, invading the lung tissue and establishing metastatic nodules in the lungs (5). Various novel improvements have been established for the treatment of patients with osteosarcoma, but metastatic disease diagnosis has not been well-studied $(6,7)$. This is due to the poor understanding of the molecular basis of osteosarcoma metastasis genes. The identification of candidate genes in the osteosarcoma metastasis process is significant, so that these may be utilized as molecular markers for diagnosis of the disease in the initial stages. In addition, targeted therapy of key metastatic molecules by selectively blocking the migratory and invasive ability of the osteosarcoma cells may present a potential strategy for the inhibition of tumor metastasis (5). The present study aimed to identify osteosarcoma metastasis genes and examine the functionality of their target genes.

\section{Materials and methods}

Patients and tumor sample collection. Osteosarcoma samples were collected from patients at the Sichuan Cancer Hospital (Chengdu, China) through an institutional review board approval committee. In addition, the project was approved by the ethics committee of Sichuan Cancer Hospital, and written informed consent was obtained from the patients. A total of 31 samples were included in the present study, which were obtained from 31 patients, including 19 males and 12 females. The age of the patients ranged from 8 to 23 years. The collected samples were separated into two categories: Osteosarcoma without metastasis and osteosarcoma with metastasis. Control serum and bone samples and distal femur biopsy specimens, which had been previously collected and stored at the hospital from healthy organ donors were used. Complete information regarding the patients and samples is presented in Table I. Immediately following collection, the tumor specimen samples were snap frozen in liquid nitrogen and stored at $-80^{\circ} \mathrm{C}$ prior to RNA extraction. All biopsy tumor specimens were confirmed to contain $>90 \%$ tumor cells. In addition, blood samples were also collected from the 31 patients and stored accordingly for additional experiments.

RNA extraction. Total RNA was extracted from the two categories of samples using the TRIzol reagent method (Invitrogen Life Technologies, Carlsbad, CA, USA), according to the manufacturer's instructions. Osteosarcoma samples were 
Table I. Patient and sample information.

A, Osteosarcoma patients without metastasis

\begin{tabular}{|c|c|c|c|c|c|}
\hline Serial No. & Patient ID & Gender & Age, years & Site & Type \\
\hline I & OS0012 & Male & 8 & Distal femur & Biopsy \\
\hline II & OS0057 & Female & 12 & Distal femur & Biopsy \\
\hline III & OS0059 & Male & 23 & Femur & Biopsy \\
\hline IV & OS0073 & Male & 14 & Tibia & Biopsy \\
\hline V & OS0079 & Female & 9 & Femur & Biopsy \\
\hline VI & OS0082 & Male & 17 & Distal femur & Biopsy \\
\hline VII & OS00123 & Male & 21 & Femur & Biopsy \\
\hline VIII & OS00138 & Female & 22 & Distal femur & Biopsy \\
\hline IX & OS00152 & Female & 11 & Femur & Biopsy \\
\hline$X$ & OS00166 & Female & 19 & Distal femur & Biopsy \\
\hline XI & OS00172 & Male & 9 & Femur & Biopsy \\
\hline XII & OS00177 & Female & 13 & Tibia & Biopsy \\
\hline XIII & OS00185 & Male & 23 & Distal femur & Biopsy \\
\hline XIV & OS00191 & Male & 14 & Distal femur & Biopsy \\
\hline XV & OS00193 & Male & 19 & Distal femur & Biopsy \\
\hline XVI & OS00199 & Female & 20 & Tibia & Biopsy \\
\hline XVII & OS00207 & Male & 15 & Distal femur & Biopsy \\
\hline
\end{tabular}

$\mathrm{B}$, Osteosarcoma patients with metastasis

\begin{tabular}{llllr}
\hline Serial No. & Patient ID & Gender & Age, years & Site \\
\hline XVIII & OS0018a & Male & 21 & Tibia \\
XIX & OS0025a & Male & 13 & Distal femur \\
XX & OS0029a & Female & 17 & Femur \\
XXI & OS0033a & Male & 10 & Distal femur \\
XXII & OS0041a & Female & 22 & Femur \\
XXIII & OS0047a & Male & Biopsy \\
XXIV & OS0049a & Male & 9 & Distal femur \\
XXV & OS0051a & Female & 15 & Distal femur \\
XXVI & OS0090a & Female & Biopsy \\
XXVII & OS00101a & Male & 20 & Biopsy \\
XXVIII & OS00145a & Male & 22 & Fiopsy \\
XXIX & OS00159a & Female & Femur \\
XXX & OS00180a & Male & 16 & Femur \\
XXXI & OS00202a & Male & 14 & Distal femur \\
\end{tabular}

ID, identification.

homogenized in TRIzol reagent using $1 \mathrm{ml}$ per 50-100 mg tissue. RNA concentrations and quality were determined using a NanoDrop 2000 spectrophotometer (Thermo Fisher Scientific, Wilmington, DE, USA), and the RNA integrity was assessed by agarose gel electrophoresis (Bio-Rad Laboratories, Inc., Hercules, CA, USA). Purification of poly A+ messenger RNA (mRNA) from the total RNA was conducted using the Qiagen Oligotex mRNA Mini kit (Qiagen, Venlo, Netherlands) according to the manufacturer's instructions. The isolated poly $\mathrm{A}+\mathrm{mRNA}$ was stored at $-80^{\circ} \mathrm{C}$ in a deep freezer for further experiments.
Complementary DNA (cDNA) synthesis and subtraction. The purified mRNA from the two groups of patients was subjected to cDNA subtraction analysis using the DsDD cDNA Subtraction and cloning kit (294-62001; Wako Pure Chemical Industries, Ltd., Osaka, Japan). The experiments were performed according to the manufacturer's instructions, which selectively amplifies differentially expressed sequences. These sequences were specific for osteosarcoma metastasis and were subsequently cloned using the DsDD cDNA Subtraction and cloning kit, as per the manufacturer's protocol. The osteosarcoma metastasis clones were sequenced 


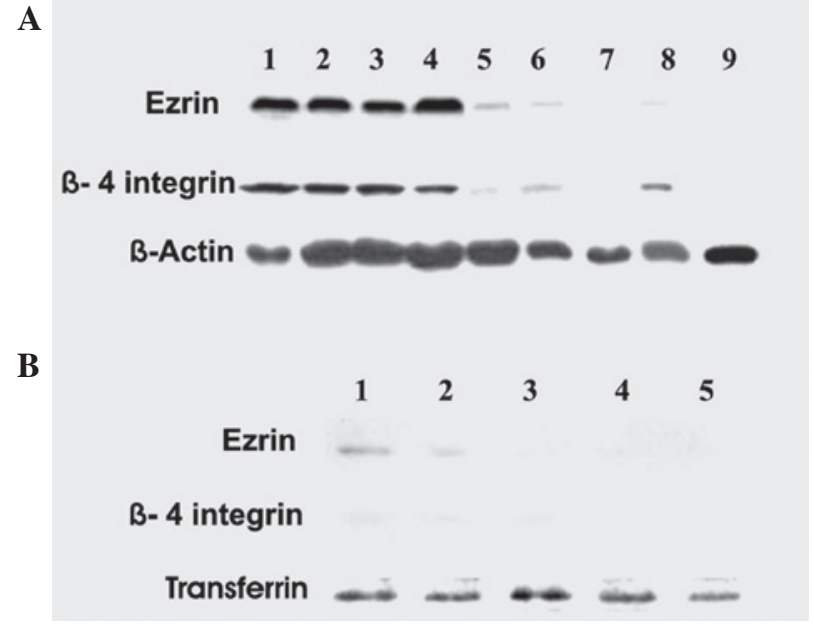

Figure 1. Validation of osteosarcoma metastasis genes. (A) Western blot analysis of ezrin and $\beta 4$ integrin expression in samples of osteosarcoma with and without metastasis and control. Lanes: 1-4, osteosarcoma with metastasis patient samples; 5-8, osteosarcoma without metastasis patient samples; 9 , control. (B) Western blot analysis of ezrin and $\beta 4$ integrin expression pattern in serum samples of osteosarcoma with and without metastasis, and control. Lanes: 1,2, osteosarcoma with metastasis patient serum samples; 3,4, osteosarcoma without metastasis patient serum samples; 5, control.

and the sequence data were subjected to Basic Local Alignment Search Tool (BLAST; http://blast.ncbi.nlm.nih.gov/Blast. cgi) analysis with the Nucleotide and Expressed Sequence Tag databases by performing the sequence alignment.

Immunoblotting. Protein samples were prepared from the two groups of patient specimens and controls, and resolved by $8 \%$ SDS-PAGE (Bio-Rad Laboratories, Inc.). The resolved protein samples from the SDS-PAGE gel were transferred to a polyvinylidene difluoride membrane (Bio-Rad Laboratories, Inc.), blocked with $4 \%$ bovine serum albumin (Sigma-Aldrich, St. Louis, MO, USA) for $1 \mathrm{~h}$ and incubated with monoclonal mouse anti-ezrin (catalog no. E8897; dilution, 1:3,000) and polyclonal rabbit anti- $\beta 4$ integrin (catalog no. SAB4501588; dilution, 1:8,000) primary antibodies (Sigma-Aldrich) overnight at $4^{\circ} \mathrm{C}$. For loading controls, monoclonal mouse anti- $\beta$-actin (catalog no. ab6276; dilution, 1:12,000) and anti-transferrin (catalog no. ab769; dilution, 1:12,000) antibodies were used (Abcam, Cambridge, UK). The nonspecific binding of the primary antibody was washed out with a 1X TBST buffer (Sigma-Aldrich). Horseradish peroxidase-conjugated polyclonal goat antimouse IgG secondary antibody (catalog no. ab97023; Abcam) was used at a dilution of 1:10,000 (incubation, $1 \mathrm{~h}$ at room temperature). The washed membrane was developed with the diaminobenzidene $/ \mathrm{H}_{2} \mathrm{O}_{2}$ substrate (Amresco LLC, Solon, $\mathrm{OH}$, USA) according to the manufacturer's instructions to produce a brown-colored product, which was detected on the membranes. Experiments were repeated at least twice to confirm the results.

\section{Results}

Observation of osteosarcoma samples. Osteosarcoma is the eighth most common cancer among children (1). Accurate diagnosis of osteosarcoma metastasis is an important requirement for effective treatment of the disease prior to metastasis. To date, molecular markers for the diagnosis of osteosarcoma metastasis are not well established. Identification of osteosarcoma metastasis genes will facilitate the design of novel molecular markers for the early detection of osteosarcoma metastasis in its initial stage. Therefore, the present project was designed to identify osteosarcoma metastasis genes. Samples were collected from 31 patients and grouped into two categories: Osteosarcoma without metastasis and osteosarcoma with metastasis. The complete information regarding the patients and samples are presented in Table I. All biopsy tumor specimens were observed and confirmed by histological analysis by a pathologist and specialized scientist, by the observation of $>90 \%$ tumor cells.

Identification of osteosarcoma metastasis genes. In order to identify the osteosarcoma metastasis genes, samples from osteosarcoma patients with and without metastasis were subjected to RNA extraction followed by cDNA subtraction using a DsDD cDNA subtraction kit. In the cDNA subtraction experiment, samples from patients with osteosarcoma without and with metastasis functioned as a driver cDNA library and tester cDNA library, respectively. The tester and driver cDNA libraries were critical for the identification of osteosarcoma metastasis genes using cDNA subtraction protocols. According to the manufacturer's instructions, the differentially expressed specific transcripts were amplified through polymerase chain reaction. Subsequently, the specific transcripts were cloned and sequenced. In total, $184 \mathrm{cDNA}$ subtracted clones were obtained. The sequenced data were analyzed by BLAST of the subtracted cDNA sequences with the EST and Nucleotide databases of the National Center for Biotechnology Information. Data pertaining to 12 of the differentially expressed specific transcripts (osteosarcoma metastasis genes), selected based upon interest, expression pattern and literature survey for further analysis, are presented in Table II.

Validation of osteosarcoma metastasis genes. In order to validate the cDNA subtraction data and study the functional aspects of the identified osteosarcoma metastasis genes, immunoblot analysis was performed for the selected targets. Based upon the results of the literature survey, ezrin and $\beta 4$ integrin were selected for the functional studies as effective osteosarcoma metastasis genes. The tissue samples from osteosarcoma patients without and with metastasis were subjected to protein sample preparation, followed by immunoblotting with anti-ezrin and anti- $\beta 4$ integrin antibodies. Analogous experiments were performed on the control tissues. As shown in Fig. 1A, the immunoblotting results illustrated that enhanced ezrin and $\beta 4$ integrin expression was detected in samples from osteosarcoma with metastasis patients, compared with the limited expression in samples of osteosarcoma without metastasis. Similar results were obtained for all 31 samples analyzed.

To further evaluate the expression pattern of ezrin and $\beta 4$ integrin in the serum samples of patients with osteosarcoma with and without metastasis, as well as the controls, protein samples were prepared and immunoblotting was performed. The data are presented in Fig. 1B, which exhibits the limited expression of ezrin and $\beta 4$ integrin detected in serum samples from osteosarcoma with metastasis patients. Limited expression of ezrin and $\beta 4$ integrin was also detected in three osteosarcoma patients without metastasis 
Table II. Osteosarcoma metastasis genes.

Osteosarcoma metastasis genes

Functions

Smad/SMAD

RANKL

Ezrin/EZR

IL-8/IL8

$\beta 4$ integrin/ ITGB4

CLIC5

NSE2

RUNX2

TGF- $\beta$

MAPK

OPN/SPP1

tp53/TP53
Responsible for the transduction of extracellular signals from transforming growth factor $\beta$ ligands to the nucleus, thereby activating downstream gene transcription $(8,9)$.

RANKL, encoded by the TNFSF11 gene $(10,11)$, functions as ligand for osteoprotegerin and a key factor for osteoclast differentiation and activation. Ezrin, also known as cytovillin or villin-2, is encoded by the EZR gene (12) and functions as a metastatic regulator (13).

A chemokine, encoded by the IL8 gene (14), which induces chemotaxis and phagocytosis.

Also known as cluster of differentiation 104, and functions as a receptor for the laminins.

Involved in chloride ion transport within various sub-cellular compartments. A component of the Smc5-6 complex, which is a SUMO ligase required for DNA damage response (15).

Also known as core-binding factor subunit $\alpha-1$, is a key transcription factor associated with osteoblast differentiation (16).

TGF- $\beta$ has crucial roles in tissue regeneration, cell differentiation, embryonic development, and regulation of the immune system.

MAPKs regulate proliferation, gene expression, differentiation, mitosis, cell survival and apoptosis (17).

OPN, also known as bone sialoprotein I, encoded by the SPP1 gene, has roles in biomineralization, bone remodeling and as an anti-apoptotic factor $(18,19)$.

Also known as cellular tumor antigen p53, phosphoprotein p53 or tumor suppressor p53, encoded by the TP53 gene, and regulates the cell cycle and functions as a tumor suppressor (20).

RANKL, receptor activator of nuclear factor $\kappa B$ ligand; IL, interleukin; CLIC5, chloride intracellular channel protein 5; RUNX2, Runt-related transcription factor 2 ; TGF- $\beta$, transforming growth factor- $\beta$; MAPK, mitogen-activated protein kinase; OPN, osteopontin.

(Patient IDs OS00152, OS00191 and OS00207). This was in comparison to the total lack of ezrin and $\beta 4$ integrin expression in the serum samples of the remaining osteosarcoma patients without metastasis and the control samples.

\section{Discussion}

Osteosarcoma metastasis is one of the most dangerous diseases among the youth population (1). Various novel improvements have been established for the treatment of patients with osteosarcoma; however the diagnosis of metastasis has not been well studied $(6,7)$. In order to detect osteosarcoma metastasis at an early stage to facilitate the effective treatment and management of the disease, the identification of genes underlying osteosarcoma metastasis is essential. In the present study, a novel technique, namely specific cDNA subtraction, was used for the identification of osteosarcoma metastasis genes. These studies emphasize the important contribution of the identification of osteosarcoma metastasis genes to the diagnosis of metastatic osteosarcoma at its initial stages.

Following cDNA subtraction, a total of 184 clones were identified, which were subsequently sequenced and analyzed using bioinformatics tools. Putative and partial sequences were also detected in the 184 clones. Among the presented 12 transcripts, ezrin and $\beta 4$ integrin were selected for further analysis, based upon interest, expression pattern and literature survey. The data from the cDNA subtraction analysis demonstrated the expression of ezrin and $\beta 4$ integrin in the samples from osteosarcoma patients with metastasis. In order to validate these results, immunoblot analysis was performed. The results confirmed that the two genes, ezrin and $\beta 4$ integrin, were osteosarcoma metastasis genes.

To further verify these data, serum samples were prepared from patients with osteosarcoma with and without metastasis and controls and immunoblot analyses were performed. Ezrin and $\beta 4$ integrin expression was detected in the samples of osteosarcoma with metastasis patients, confirming that these were osteosarcoma metastasis genes. By contrast, limited expression of ezrin and $\beta 4$ integrin was also detected in certain osteosarcoma without metastasis patients. This may indicate that these patients are under risk of osteosarcoma metastasis at initial stage. Except in these three patients, no expression of ezrin and $\beta 4$ integrin was observed in samples from osteosarcoma patients without metastasis. These results suggested that the presence of ezrin and $\beta 4$ integrin may be utilized for the diagnosis of osteosarcoma metastasis in initial stages.

Ezrin is a member of the ezrin/radixin/moesin (ERM family), which shares the common membrane-binding N-terminal FERM domain with band-4.1 family members $(21,22)$. Ezrin has a role in the metastatic spread 
of not only osteosarcoma, but also mammary and pancreatic adenocarcinomas (23-25). Notably, in the present study, the expression of ezrin in the serum of patients with osteosarcoma with metastasis and the limited expression in patients with osteosarcoma without metastasis highlighted the fact that ezrin may be employed as a marker for osteosarcoma metastasis. Further studies are essential for the development of ezrin as a novel marker for the detection of osteosarcoma metastasis at early stages.

Integrins consist of non-covalently associated $\alpha$ and $\beta$ subunits. These heterodimers regulate key biological processes, including cell adhesion, signaling, migration, proliferation, survival, angiogenesis, oncogenesis and metastasis (26-30). To date, $18 \alpha$ subunits and $8 \beta$ subunits have been identified. The subunit $\alpha 6-\beta 4$ (known as $\beta 4$ integrin) was originally identified as a tumor-associated antigen. It was also demonstrated that the expression and function of $\beta 4$ integrin was implicated in the progression of various types of cancer (31-34). Of note, the results of the present study confirmed the presence of $\beta 4$ integrin expression in patients with osteosarcoma with metastasis, which indicated that $\beta 4$ integrin may be an osteosarcoma metastasis gene. In addition, limited expression of $\beta 4$ integrin was observed in certain samples of patients with osteosarcoma without metastasis, which may indicate those patients underrisk of osteosarcoma metastasis, highlighting the efficacy of this potential marker.

In conclusion, the results of the present study indicated that ezrin and $\beta 4$ integrin were highly expressed in patients with osteosarcoma metastasis. Based upon the cDNA subtraction analysis followed by immunoblotting experiments, it was concluded that these two transcripts were osteosarcoma metastasis genes. Further studies are required to develop $\beta 4$ integrin and/or ezrin as novel markers for the detection of osteosarcoma metastasis in the initial stages.

\section{Acknowledgements}

The present study was financially supported by Sichuan Province Applied Basic Research Project (no. 2013JY0152). The authors would like to thank the patients, whose support provided the backbone for the project. The authors also wish to thank the institutional review board approval committee and ethics committee for facilitating the successful completion of this project.

\section{References}

1. Man TK, Chintagumpala M, Visvanathan J, et al: Expression profiles of osteosarcoma that can predict response to chemotherapy. Cancer Res 65: 8142-8150, 2005.

2. Ottaviani G and Jaffe N: The epidemiology of osteosarcoma. Cancer Treat Res 152: 3, 2009.

3. Dorfman HD and Czerniak B: Bone cancers. Cancer 75: 203-210, 1995.

4. Pratap J, Lian JB, Javed A, Barnes GL, Van Wijnen AJ, Stein JL and Stein GS: Regulatory roles of Runx 2 in metastatic tumor and cancer cell interactions with bone. Cancer Metastasis Rev 25: 589-600, 2006.

5. Wan X, Kim SY, Guenther LM, et al: Beta4 integrin promotes osteosarcoma metastasis and interacts with ezrin. Oncogene 28 : 3401-3411, 2009.

6. Meyers PA, Heller G, Healey JH, Huvos A, Applewhite A, Sun M, and LaQuaglia M: Osteogenic sarcoma with clinically detectable metastasis at initial presentation. J Clin Oncol 11: 449-453, 1993.

7. Harris MB, Gieser P, Goorin AM, et al: Treatment of metastatic osteosarcoma at diagnosis: A Pediatric Oncology Group Study. J Clin Oncol 16: 3641-3648, 1998.
8. Heldin CH, Miyazono K and ten Dijke P: TGF-beta signalling from cell membrane to nucleus through SMAD proteins. Nature 390: 465-471, 1997.

9. Wrana JL: Crossing Smads. Science STKE 2000: re1, 2000.

10. Anderson DM, Maraskovsky E, Billingsley WL, et al: A homologue of the TNF receptor and its ligand enhance T-cell growth and dendritic-cell function. Nature 390: 175-179, 1997.

11. Wong BR, Rho J, Arron J, et al: TRANCE is a novel ligand of the tumor necrosis factor receptor family that activates c-Jun N-terminal kinase in T cells. J Biol Chem 272: 25190-25194, 1997.

12. Gould KL, Bretscher A, Esch FS and Hunter T: cDNA cloning and sequencing of the protein-tyrosine kinase substrate, ezrin, reveals homology to band 4.1. EMBO J 8: 4133-4233, 1989.

13. Yu Y, Khan J, Khanna C, Helman L, Meltzer PS and Merlino G: Expression profiling identifies the cytoskeletal organizer ezrin and the developmental homeoprotein Six-1 as key metastatic regulators. Nat Med 10: 175-181, 2004.

14. Modi WS, Dean M, Seuanez HN, Mukaida N, Matsushima K and O'Brien SJ: Monocyte-derived neutrophil chemotactic factor (MDNCF/IL-8) resides in a gene cluster along with several other members of the platelet factor 4 gene superfamily. Hum Genet 84 : 185-187, 1990.

15. Andrews EA, Palecek J, Sergeant J, Taylor E, Lehmann AR and Watts FZ: Nse2, a component of the Smc5-6 complex, is a SUMO ligase required for the response to DNA damage. Mol Cell Biol 25: 185-196, 2005.

16. Green RE, Krause J, Briggs AW, et al: A draft sequence of the Neandertal genome. Science 328: 710-722, 2010.

17. Pearson G, Robinson F, Beers Gibson T, et al: Mitogen-activated protein (MAP) kinase pathways: Regulation and physiological functions. Endocr Rev 22: 153-183, 2001.

18. Denhardt DT, Noda M, O'Regan AW, Pavlin D and Berman JS: Osteopontin as a means to cope with environmental insults: Regulation of inflammation, tissue remodeling, and cell survival. J Clin Invest 107: 1055-1061, 2001.

19. Standal T, Borset M and Sundan A: Role of osteopontin in adhesion, migration, cell survival and bone remodeling. Exp Oncol 26: 179-184, 2004.

20. Kern SE, Kinzler KW, Bruskin A, Jarosz D, Friedman P, Prives C and Vogelstein B: Identification of p53 as a sequence-specific DNA-binding protein. Science 252: 1708-1711, 1991.

21. Bretscher A, Edwards K, and Fehon RG: ERM proteins and merlin: Integrators at the cell cortex. Nat Rev Mol Cell Biol 3: 586-599, 2002.

22. Louvet-Vallée S: ERM proteins: From cellular architecture to cell signaling. Biol Cell 92: 305-316, 2000.

23. Khanna C, Khan J, Nguyen P, et al: Metastasis-associated differences in gene expression in a murine model of osteosarcoma. Cancer Res 61: 3750-3759, 2001.

24. Khanna C, Wan X, Bose S, et al: The membrane-cytoskeleton linker ezrin is necessary for osteosarcoma metastasis. Nat Med 10: 182-186, 2004.

25. Nestl A, Von Stein OD, Zatloukal K, Thies WG, Herrlich P, Hofmann M and Sleeman JP: Gene expression patterns associated with the metastatic phenotype in rodent and human tumors. Cancer Res 61: 1569-1577, 2001.

26. Aplin AE, Howe AK and Juliano R: Cell adhesion molecules, signal transduction and cell growth. Curr Opin Cell Biol 11: 737-744. 1999.

27. Giancotti FG and Ruoslahti E: Integrin signaling. Science 285: 1028-1033, 1999.

28. Hood JD and Cheresh DA: Role of integrins in cell invasion and migration. Nat Rev Cancer 2: 91-100, 2002.

29. Howe A, Aplin AE, Alahari SK and Juliano R: Integrin signaling and cell growth control. Curr Opin Cell Biol 10: 220-231, 1998.

30. Hynes RO: Integrins: Bidirectional, allosteric signaling machines. Cell 110: 673-687, 2002.

31. Guo W, Pylayeva Y, Pepe A, Yoshioka T, Muller WJ, Inghirami G and Giancottin FG: Beta 4 integrin amplifies ErbB2 signaling to promote mammary tumorigenesis. Cell 126: 489-502, 2006.

32. Mariotti A, Kedeshian PA, Dans M, et al: EGF-R signaling through Fyn kinase disrupts the function of integrin alpha6beta4 at hemidesmosomes: Role in epithelial cell migration and carcinoma invasion. J Cell Biol 155, 447-458, 2001.

33. Nikolopoulos SN, Blaikie P, Yoshioka T, Guo W and Giancotti FG: Integrin beta4 signaling promotes tumor angiogenesis. Cancer Cell 6: 471-483, 2004.

34. Trusolino L, Bertotti A and Comoglio PM: A signaling adapter function for alpha6beta4 integrin in the control of HGF-dependent invasive growth. Cell 107: 643-654, 2001. 